\title{
On Planetary Electromagnetism and Gravity
}

\author{
Ashwini Kumar Lal \\ Town \& Country Planning Organisation, New Delhi, India \\ E-mail:ashwinikumar_lal@yahoo.co.in \\ Received January 15, 2011; revised March 9, 2011; accepted March 19, 2011
}

\begin{abstract}
Study of the interiors of the various terrestrial planets, as presented in the paper, leads to the possibility of planetary gravity being linked to the electromagnetism generated in the planetary interiors. Findings of the study undertaken suggest that Earth's gravitational attraction may be attributed to magnetic coupling experienced between Earth's electromagnetism and all the earthly objects-electrically charged or uncharged. More precisely, terrestrial gravity is deemed to be outcome of the bound state of the planetary electromagnetism.
\end{abstract}

Keywords: Electromagnetism, Gravity, Planetary Dynamo, Coriolis Force, Earth, Venus, Mars, Mercury

\section{Introduction}

Jordan and Stevenson et al. [1-3] provide reasonable models of the interiors of the terrestrial planets, based on analysis of the latest available physical data in respect of the various parameters influencing composition of the planetary interiors. The basic assumptions of all their models are that the core dynamos are driven by thermal and/or chemical convention, that radiogenic heat production is confined to the mantle, and that any inner core (IC) that may nucleate excludes the light alloying component ( $\mathrm{S}$ or $\mathrm{O}$ ) which mixes uniformly upward through the outer core (OC). The assumption that the cores contain no radioactive heat sources implies that convective motions necessary to drive a dynamo must be generated either by secular cooling of an entirely fluid OC or by IC solidification. All models assume whole mantle convection. Stevenson et al. found that small changes in the model parameters can result in completely fluid, non-convecting cores (without dynamo action), convecting fluid OCs with IC growth (with dynamo action), and almost solid cores with only thin OC fluid shells now remaining (and preferably with no dynamo).

\section{Earth}

It has been known since 1600 A.D. that the Earth as a whole is a dipole magnet. Most of the planets, however, are too hot for any magnetic material to retain its magnetism. Temperature within the earth increases with depth at an average rate of $30^{\circ} \mathrm{C}$ per $\mathrm{km}$, while the Curie points for iron-bearing minerals are $<800^{\circ} \mathrm{C}$. Furthermore, permanently magnetized minerals cannot move about rapidly enough to account for the changes in the strength, direction and pattern of the Earth's magnetic field [3]. Interiors of Earth (Figure 1) are probed indirectly using the time-travel of refracted and reflected seismic waves generated by earthquakes. Seismic waves namely, Primary $(\mathrm{P})$ or compression waves and Secondary (S) or Shear waves and others transmitted within the interiors of the Earth provide important clues about the planet's internal structure (Figure 2). Seismic measurements show that the Earth is primarily divided into four main layers: crust, mantle, outer core, and inner core. The outermost layer, crust, composed mainly of aluminosilicates, has a variable thickness being $35-70 \mathrm{~km}$ in the continents, and $5-10 \mathrm{~km}$ thick in ocean basins. The mantle is the layer beneath the crust that extends half way to the centre. It is mainly composed of ferro-magnesium silicates, and is about $2900 \mathrm{~km}$ thick. At $1000^{\circ} \mathrm{C}$, mantle is mostly in plastic state. The next layer is $3500 \mathrm{~km}$ thick core constituting about $55 \%$ of the Earth's interior, which is separated into two distinct layers-2300 km thick liquid outer core and $1200 \mathrm{~km}$ thick solid inner core. The most salient feature of the core is that it never transmits $\mathrm{S}$-waves. Since failure to transmit and transverse waves is a distinctive characteristic of liquids, it is generally accepted that the core material is in a liquid state, mainly of molten iron with about $10 \%$ sulfur and/or oxygen (Fe-S-O mix), while the inner core is almost entirely composed of solid iron. At the inner core, where the temperature reaches incredible level of $3,700^{\circ} \mathrm{C}$, excessively high pressure of $360 \mathrm{GPa}$ prohibits the inner core material to be in liquid state. 


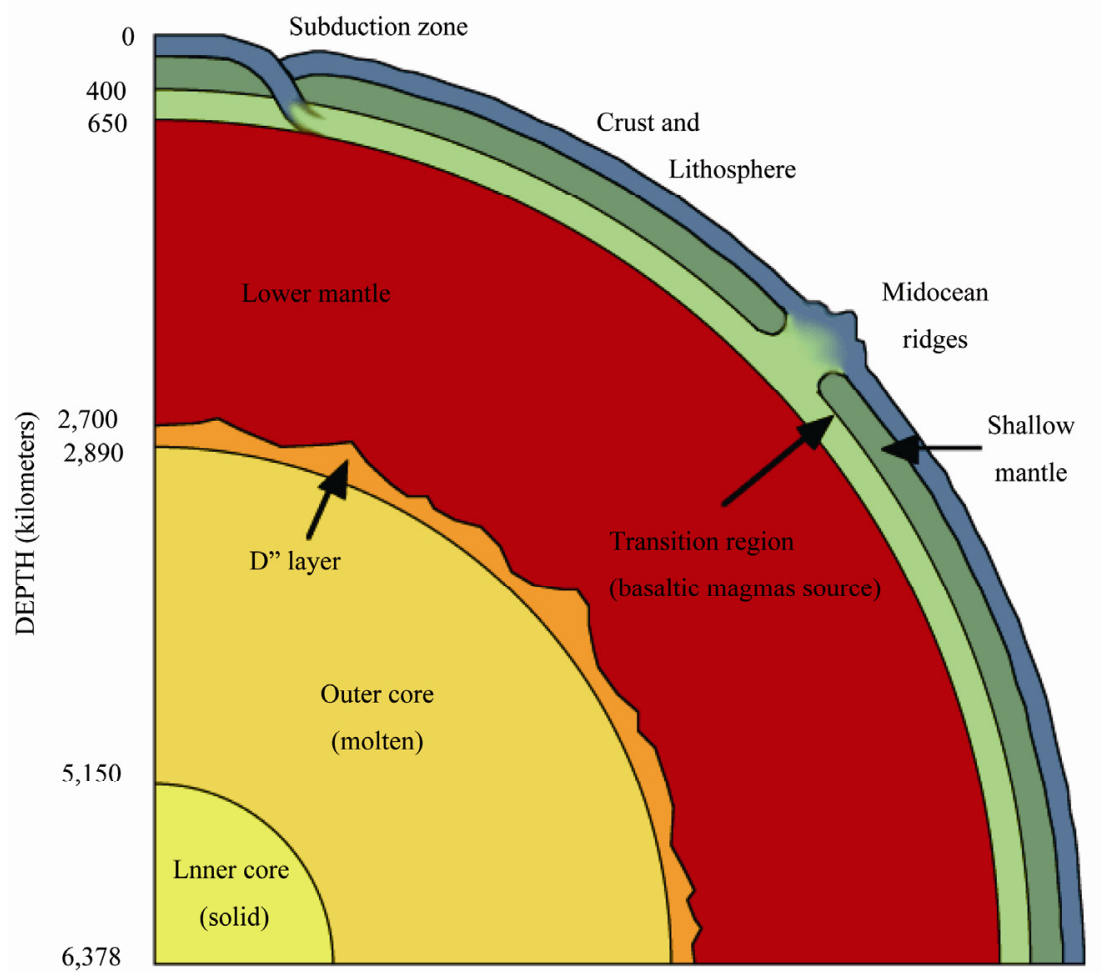

Figure 1. Internal structure of Earth adapted from: J. K. Beatty and A. Chaikin, eds, The New Solar System, Massachusetts, Sky Publishing, 3rd Edition, 1990.

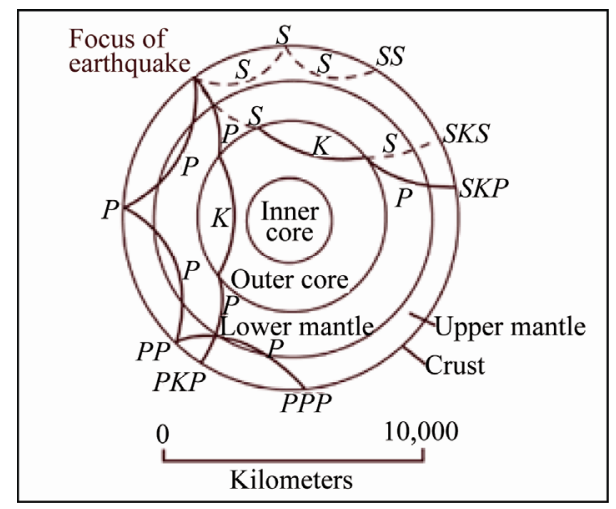

Figure 2. Cross section of the whole Earth, showing the complexity of paths of earthquake waves. The paths curve because the different rock types found at different depths change the speed at which the waves travel. Solid lines marked $P$ are compressional waves; dashed lines marked $S$ are shear waves. $S$ waves do not travel through the core but may be converted to compressional waves (marked $K$ ) on entering the core $(P K P, S K S)$. Waves may be reflected at the surface $(P P, P P P, S S)$. Adapted from: http://pubs.usgs.gov/ gip//interior/

\section{Electromagnetism within Earth}

Origin of Earth's magnetic field is generally attributed to Coriolis force resulting from Earth's rotation and generation of self-exciting dynamo in the electrically conducting outer fluid core in view of the observed secular variations in the planet's magnetic field [3-6]. Stacey suggests that if the light alloying element in the core is sulfur, and if this is confined to the OC, the IC may be mainly of iron and nickel $[7,8]$. With the possibility of impurities inherent in the IC material, there is always probability of generation of electromotive force within the inner core as well. The process of generation of electromagnetism within the IC, however, is very complex. Presence of $\mathrm{Fe}$ and $\mathrm{Ni}$ at varying high temperature and pressure conditions within the IC might also lead to generation of electromotive force within Earth's IC due to seebeck or thermoelectric effect [9] which could subsequently lead to generation of electromagnetism within the Earth's IC. Flow of electric currents in the IC might have been triggered also by the influence of the fluctuating electromagnetic field in the adjoining OC. Thus, within Earth's interiors, electromagnetism is not only generated in the outer core, but it is also produced in the inner core. Planetary dynamo [6] mostly appears to operate with an internal field $\sim(2 \rho \Omega / \sigma)^{1 / 2}$, where $\rho$ is the fluid density, $\Omega$ is the planetary rotation rate, and $\sigma$ is the conductivity (SI Units).

\section{Mechanism of Gravitational Pull}

Earth's gravity, it appears, is intrinsically related to the generation of tremendous electromagnetic force within 
the convecting fluid in the outer core. A probe into the interiors of the Earth $[5,6,10,11]$ indicates that there is perhaps no factor other than the generation of electromagnetism within the planetary interior that is responsible for the pulling action of Earth exerted over worldly objects. The giant electromagnet seated within the Earth is bounded by the layers of mantle and crust. As such, the intensity of magnetic field generated due to dynamo action in the Earth's core gets depleted due to the presence of overlaying layers of mantle and crust comprising mostly of silicates and aluminates. This is the reason why the intensity of Earth's magnetic field ( $\sim 1$ gauss) experienced on its outer surface is very feeble, rather it is several hundred times weaker than the field between the ends of a toy horseshoe magnet despite Earth retaining its magnetic property. The situation is somewhat similar to the one in which a ferromagnet surrounded by a non-ferrous material still demonstrates pulling effect over magnetic substance like iron needle brought in its proximity. So is the case with Earth's electromagnet. In view of this phenomenon, it could be inferred that gravity of Earth is possibly manifestation of electromagnetism generated in Earth's interiors. Similar is perhaps true also about other terrestrial planets like Venus, Mars, and Mercury.

Effect of gravity on a body is analogous to the action of a magnet on a ferrous material. As regards the mutual interaction between Earth's gravity and any worldly object, the pull experienced on the object due to Earth's gravity is perhaps nothing but interaction between the electromagnetism of the Earth and the magnetism of that object. As per the findings of the quantum physics, all ordinary substances (charged or uncharged) do possess some magnetic property though, in some case, it may be very feeble (a thousand to a million times less) compared to the effect observed in ferromagnetic materials [12]. The Earth's giant electromagnet apparently pulls every object towards its centre with the same intensity as revealed by the Galileo's experiment. It is so because of its being immensely powerful compared to the magnetism possessed by the various worldly objects. This is possibly the reason, everything appears to be falling under Earth's gravitational influence with equal intensity. In view of the above, the pull due to the Earth's gravity experienced by any earthly object is equivalent to the magnetic coupling (interaction) between the Earth's giant magnetism and the magnetism of that object. This is probably the mechanism of the earth's gravitational pull.

\section{Venus}

Since Venus has about the same size and average density as the Earth, it is quite probable that it does possess a metallic core with internal temperatures similar to those in Earth. Lewis [11] suggests that Venus has a massive core of Fe-Ni alloy surrounded by a mantle of $\mathrm{Fe}_{2}{ }^{++}$free magnesium silicates. The lower density of Venus, as compared to Earth's, indicates that it might have a smaller core than Earth's. It is also believed, the Venusian core has less sulfur content than Earth's. No probe sent to Venus has yet detected a magnetic field; if one exists, it is at least 10,000 times weaker than Earth's. Assuming that Venus and Earth have similar moments of inertia, Toksoz and Johnston [13] have estimated that Venus has a completely separated Fe-Ni core of radius $2900 \mathrm{~km}$. Stevenson et al. [2] predict a stably stratified, completely fluid core likely present state for Venus. The absence of a significant IC in Venus is probably due to its slightly higher temperature and lower central pressure relative to Earth (290 Gpa of Venus compared to Earth's 360 Gpa). Such a core is unlikely to generate magnetic field. However, according to Stevenson [6], though Coriolis force is dynamically important, slow rotation of a planet may be more favourable for a dynamo than the scenario for fast rotation. This aspect favours functioning of the electrical dynamo also within the Venusian core despite the fact that rotational speed of Venus is much less compared to Earth (243 days of Venus against Earth's 24 hours). Besides, with the presence of stratified electrically conducting materials at varying high temperature and pressure conditions within the Venusian core, there is all likelihood of generation of electromotive force within the core due to seebeck or thermoelectric effect which could lead to generation of electromagnetism within the core. Moreover, the electrically conducting materials within the Venusian core is always likely to contain some impurities which could also trigger generation of electromotive force within the electrically conducting Venusian core. Combination of electric currents and generation of electromotive force within the core is an ideal case for generation of electromagnetism within the as the most Venusian core.

\section{Mars}

Mars admits all possibilities for the state of its core. Stevenson et al. [2] favour a completely fluid core, since it predicts for cosmochemically plausible sulfur content of $15 \%$ or more by weight, and also provides an explanation for the absence of substantial magnetic field. In 2003, researchers at NASA's Jet Propulsion Laboratory [14], after analyzing three years of radio tracking data from the Mars Global Surveyor spacecraft, had reported in Science that Mars has not cooled to a completely solid iron core, rather its interior is made up of either a completely liquid core with a solid inner core. Scientists had earlier seen no signs of contemporary magnetic field in data from Mars, leading them to write off the planet as geologically dead. The 2003 findings about the Martian interiors corrobo- 
rated Stevenson's prediction [15] made in 2001 regarding Mars having a liquid, conductive outer core with a solid inner core like Earth. Stewart [16] has reported in Science that if a liquid metal moves around a solid core, it could create a dynamo like the one found in Earth's core. A functional dynamo within the Martian core along with the electrically conducting core materials at high temperature and pressure conditions within the core are, in all probability, is responsible for generation of electromagnetism within the Martian interiors very much the way electromagnetism is generated in the Earth's interiors.

\section{Mercury}

Measurements made during the Mariner 10's flybys detected that Mercury has an intrinsic magnetic field. It was inferred that the field is dominantly dipolar with an average equatorial field intensity of 0.003 gauss which corresponds to roughly $0.07 \%$ that of the Earth [17]. Mercury models suggest a large solid IC surrounded by a significant FeS layers. Thermal evolution models of Mercury demonstrate that the core can remain partially liquid depending upon the amount of sulfur present therein. Recent Earth-based radar measurements of Mercury's rotation have led to discovery of a relatively large longitudinal libration, implying that Mercury's core is at least partially molten [18]. Notably, the existence of a global magnetic field is consistent with a convection-driven dynamo in liquid outer core. Operation of Mercury's dynamo requires a liquid layer of minimum thickness of $4 \%$ of core radius only [6]. The functional dynamo along with the presence of electrically conducting materials within the interiors of Mercury produce high magnitude electromagnetism as in other terrestrial planets.

\section{Concluding Remarks}

Discussions in foregoing paragraphs lead to a very interesting probability that gravity of the terrestrial planets may be intrinsically related to the generation of electromagnetism within their interiors. In other words, gravity and electromagnetism might perhaps be two sides of the same coin with the exception that gravity is electromagnetic force of only attracting type in the light of the observed pulling effect of the gravity force resulting from confinement of planetary dynamo within the interiors of the terrestrial planets. Study of the terrestrial interiors like Earth's also suggests that gravitational attraction between Earth and any object may be attributed to magnetic coupling experienced between Earth's magnetism and the magnetism possessed by earthly objects - charged or uncharged. The observations made in the paper might help establish the true nature of the gravity force. Clues provided in the paper may also help resolve longstanding issue in science relating to unification of the fundamental forces (viz. gravity, electromagnetism, weak interaction, and strong interaction) in nature, which is of vital importance in evolving Theory of Everything (TOE).

It will be worthwhile undertaking experiments in the laboratory relating to the interaction between solid spheres of metallic and non-metallic objects to test the viability of the hypothesis proposed in the paper. Experiments, performed in many laboratories in the past, have demonstrated that electromagnetic forces can pull hot gases into distinct structures lending support to the claim made in the paper.

\section{References}

[1] T. H. Jordan, "Structural Geology of Earth's Interiors," Proceedings of the National Academy of Sciences, Vol. 76, 1979, pp. 4192-4200.

[2] D. J. Stevenson, et al., "Magnetism and Thermal Evolution of Terrestrial Planets," Icarus, Vol. 54, No. 3, 1979, pp. 466-489. doi:10.1016/0019-1035(83)90241-5

[3] D. J. Stevenson, "Planetary Magnetic Fields," Reports on Progress in Physics, Vol. 46, No. 5, 1983, pp. 555-620. doi:10.1088/0034-4885/46/5/001

[4] C. R. Carrigan, and D. Gubins, "The Source of the Earth's Magnetic Field," Scientific American, Vol. 240, No. 2, February 1979, pp. 118-129.

[5] J. A. Jacobs, "The Earth's Core," 2nd Edition, Academic, London, 1987.

[6] D. J. Stevenson, "Planetary Magnetic Fields," Earth and Planetary Science Letters, Vol. 288, No. 1-2, 2003, pp. 1-11. doi:10.1016/S0012-821X(02)01126-3

[7] F. D. Stacey, "Physical Properties of Earth's Core," Geophysical Survey, Vol. 1, 1972, pp. 99-119.

[8] F. D. Stacey, "A Thermal Model of the Earth," Physics of the Earth and Planetary Interiors, Vol. 15, No. 4, 1977, pp. 341-348. doi:10.1016/0031-9201(77)90096-6

[9] The World Book Encyclopaedia of Science, World Book, Chicago, Vol. 1, 1989.

[10] B. P., Glass, "Introduction to Planetary Geology," Cambridge University Press, Cambridge, 1978.

[11] J. S. Lewis, "Metal/Silicate Fractionation in the Solar System," Earth and Planetary Science Letters, Vol. 15, No. 3, 1972, pp. 286-290. doi:10.1016/0012-821X(72)90174-4

[12] R. P. Feynman, "The Feynman Lecture on Physics," Addison-Wesley, Massachussetts, Vol. 2, 1964.

[13] M. N. Toksoz and D. H. Johnston, "The Evolution of Moon and Terrestrial Planets", In: J. H. Pomeroy, Ed., Soviet American Conference on Cosmochemistry of the Moon and Planets, NASA Publ., Moscow, SP-370295, 1977.

[14] C. F. Yoder, et al., "Fluid Core of Mars from Detection of Solar Tide," Science, Vol. 300, No. 5617, 2003, pp. 299-304.

[15] D. J. Stevenson, "Mars Core and Magnetism," Nature, Vol. 412, No. 6843, 2001, pp. 214-219. 
[16] A. J. Stewart, "Mars: A New Core-Crystallization Regime," Science, Vol. 316, No. 5829, pp. 1323-1325. doi:10.1126/science.1140549

[17] N. F. Nees, "The Magnetic Field of Mercury," Journal of Geophysical Research, Vol. 80, No. 19, 1975, pp. 2708-
2716. doi:10.1029/JA080i019p02708

[18] J. L. Margot et al., "Large Longitude Libration of Mercury Reveals A Molten Core," Science, Vol. 316, No. 5825, 2007, pp. 710-714. doi:10.1126/science. 1140514 